\title{
Lean Enablers for Clinical Laboratories
}

\section{Bohdan W Oppenheim ${ }^{1 *}$, Michael H Kanter ${ }^{2}$, Onie Bueno ${ }^{3}$, Vincent L Dizon ${ }^{4}$, Louie M Farnacio ${ }^{5}$, Paulette L Medina $^{6}$, Mike M Moradian ${ }^{7}$, Chiemi Tabata ${ }^{8}$ and Michael S Tiffert ${ }^{9}$}

${ }^{1} \mathrm{PhD}$, Professor of Systems Engineering,

${ }^{2}$ MD, CPPS, Regional Medical Director of Quality \& Clinical Analysis, Chief Quality Officer, The Permanente Federation

${ }^{3}$ Director of Operations, Bacteriology and Molecular Microbiology, SCPMG

${ }^{4}$ MBA MT(ASCP) DLM SBB. Director of Operations, Chemistry Services

${ }^{5}$ Director of Laboratory Services,

${ }^{6}$ Assistant Director of Operations, RRL Bacteriology, Lab

${ }^{7}$ PhD, Director of Operations, Molecular Genetic Pathology Regional

${ }^{8}$ Assistant Director of Operations, RRL Automated Chemistry,

${ }^{9} \mathrm{MS}$, Section Manager, Cytogenetics, Regional Genetics Laboratory,

*Corresponding author: Dr. Bohdan W Oppenheim, Professor of Systems Engineering, Associate Director for Healthcare Systems Engineering, Loyola Marymount University, Los Angeles, USA

Submission: 悳 October 23, 2017; Published: 每 November 27, 2017

\begin{abstract}
A new intellectual product named Lean Enablers for Clinical Laboratories (LEfCL) is described. It is a collection of 136 best practices for clinical laboratory operations, containing collective wisdom on how to practice laboratory management, focusing on simultaneous improvements in test quality, productivity, laboratory capacity and stakeholder satisfaction, and reductions of test turnaround time and cost. The methodology follows an awarded method established in Systems Engineering and represents an extension of that engineering knowledge into clinical practice. The product has been developed by experienced directors from Kaiser Permanente regional clinical laboratory while pursuing a graduate Certificate in Lean Healthcare at Loyola Marymount University. LEfCL are organized into six well-known Lean Principles: Value, Value Stream Mapping, Flow, Pull, Perfection and Respect. The paper also identifies 46 Challenges that prevent success. Results of Lean improvements measured over the period of $2014-2016$ are quoted for five laboratories. The LEfCL are not intended to become a mandatory practice. Instead, they should be used as a checklist of good practices. LEfCL are formulated as a comprehensive set of practices, intended for future training of new hires and as reference material. As such, they include both basic and advanced practices.
\end{abstract}

Keywords: Lean; Lean enablers; Clinical laboratories; Process improvement; Streamlining

\section{Introduction}

The healthcare industry in the US is the largest segment of the economy; exceeding three trillion dollars [1]. While the ACA seems to have started bending the overall healthcare cost curve downward [2], most of healthcare as well as individual patients are still experiencing high costs across the industry. The rapid changes following the introduction of the ACA and recent political turbulence tend to create stress within healthcare providing entities. Clinical laboratories are no exception and are feeling the challenges of higher number of patients, lower revenues, higher costs, quality and service challenges, and increasing regulatory oversights. This paper offers a methodology to help reverse this trend in clinical laboratory operations using Lean methodology.

\section{Materials and Methods}

Lean Thinking in Healthcare, or Lean Healthcare for short, is well established as the modern work paradigm and has been widely published, e.g. in $[3,4]$. It has been proven as the systematic approach to simultaneously reduce waste, cost and process throughput times, improve the capacity and quality, provide better services, and improve the work morale of the employees and other stakeholders. Lean routinely reduces the amount of waste in the system by $30-50-70 \%$ [5]. This paper extends the paradigm to clinical laboratories.

The tool that we have developed is called Lean Enablers. A Lean Enabler is a best practice minimizing waste, test turnaround time, cost and frustrations of providers and patients served, as well as maximizing test quality and work satisfaction, and is based on Lean Thinking. Each Enabler is typically one sentence long, stated in imperative form, eg, "Reduce batch sizes". Some Enablers are described in several short related sentences. Most Enablers listed in this paper are self-explanatory; a few which may not be are amended with brief explanatory paragraphs. 
The general methodology used followed two large projects that involved six years of work by hundreds of experienced systems engineers and defense and commercial program managers from top global companies. The results have been published in [6], as well as in two books: [5,7]. In short, the methodology is based on capturing tacit knowledge and experience of experts, using intense brainstorming and comprehensive iterations and editing of the captured knowledge. Both publications received awards from the sponsoring organizations International Council on Systems Engineering (INCOSE) and Project Management Institute (PMI), as well as three awards from the Shingo Prize Institute in 2011, 2013 and 2017. While the scope of the present project developing Lean Enablers for Clinical Laboratories was significantly smaller than in the above-mentioned works (only 11 individuals working productively for about 10 weeks) fast progress was made possible by applying the former methods and lessons learned.

\section{Setting}

This study was undertaken in the Regional Laboratory of the Southern California Kaiser Permanente (KP) region. This laboratory is part of a complete laboratory system that provides services for 4.1 million members covering over 200 medical office buildings and fourteen hospitals. There are approximately 6000 physicians in the system. Patients receive essentially all their care within the KP system. The laboratory performs over 30 million tests per year and covers a broad range of routine and highly specialized clinical laboratory tests. Some routine tests and emergency room/hospital tests are performed in the local hospitals with non-stat routine and highly specialized tests being performed at the regional lab.

\section{Definitions}

Lean thinking: Three concepts are fundamental to the understanding of Lean Thinking: value, waste, and the process of creating value without waste, which is captured in the so-called six Lean Principles. These concepts are summarized in this section in the general context of Lean clinical laboratories. Any reader who is new to the concepts of Lean Thinking would greatly benefit from reading the seminal books by $[3,8]$. Lean Thinking adopts several practices previously known by other names, such as Six Sigma, total quality management, concurrent engineering, and others. The criterion we use for adoption is simple: If a best practice promotes value, reduces waste, and can be captured into the Six Lean Principles, it is called Lean.

Value: The concepts of Value and customer are interlinked. Value has a meaning only for a specific customer or groups of customers. In clinical testing laboratories both the patient and the provider (physician or nurse) who ordered the test are the external customers. They need accurate test results as soon as possible in order to diagnose the patient and provide the needed treatment. Fast treatment often saves lives and is always conducive to better health. Another aspect of value is cost. Driven by the ACA, competition, and national imperative, clinical laboratories need to strive to keep lowering the costs of their testing as long as the test quality or throughput time are not jeopardized.

Waste: The ability to identify and eliminate waste is a critical skill for Lean practitioners. All work activities are classified into the following three categories [8]:

1. Value-added (VA) activities, which must satisfy the following three conditions:

a) Transform information or material, or reduce uncertainty (cannot be an unnecessary bureaucratic task that creates no value).

b) The cost of the value is reasonable to the customer (in more complex activities, such as clinical testing, the acceptance is implicit, that is, if the customer understood the details, he or she would approve of this cost.)

c) It is done right the first time. Rework resulting from former defects does not count.

2. Required (also called necessary) non-value-added (RNVA) activities, which do not meet the previous definition, but which cannot be eliminated because they are required by regulatory agencies, law, contract, company mandate, current technology, or other similar reason, eg the HIPAA - mandated activities.

3. Non-value-added (NVA) activities, which consume resources and create no value. They are pure waste, and should be eliminated immediately. Taiichi Ohno [9] classified waste into seven (later extended to eight [5] categories shown in the left column of Table 1. The right-hand side column lists examples of waste in clinical laboratories.

Table 1: Eight Types of Waste with Examples from Clinical Laboratories.

\begin{tabular}{|l|r|}
\hline Eight Wastes & Clinical Laboratory Examples \\
\hline \multirow{4}{*}{ Overproduction } & Producing more than needed by next processes \\
\cline { 2 - 3 } & $\begin{array}{c}\text { Work that is not needed by anyone, bureaucracy } \\
\text { Redundant or unneeded tasks }\end{array}$ \\
\cline { 2 - 3 } & $\begin{array}{r}\text { Excessive paperwork, multiple databases, and duplicate procedures } \\
\text { Over-dissemination, that is sending information to too many people (e.g., } \\
\text { excessive e-mail distribution) }\end{array}$ \\
\cline { 2 - 3 } & \begin{tabular}{c} 
Lack of reuse of expertise, reinventing the wheel \\
\cline { 2 - 3 }
\end{tabular} \\
\cline { 2 - 3 } &
\end{tabular}

${ }^{1}$ This section has been adapted by the first author from chapter 3 of his book [5]. 


\begin{tabular}{|c|c|}
\hline \multirow{4}{*}{ Waiting } & Samples idle, waiting for next step \\
\hline & Large queues \\
\hline & $\begin{array}{c}\text { Humans waiting for approval or signature or information, or information } \\
\text { waiting for humans }\end{array}$ \\
\hline & Unnecessary serial effort \\
\hline \multirow{4}{*}{ Unnecessary Movement of Samples or Information } & Transporting specimens inefficiently \\
\hline & Inefficient layout of the laboratory \\
\hline & Excessively moving samples or patients through spaces \\
\hline & Excessive movement of information \\
\hline \multirow{4}{*}{ Over-Processing of Information } & Test or test equipment refinements beyond what is needed \\
\hline & Lack of standardization \\
\hline & Use of excessively complex or fragmented software \\
\hline & Inefficient "research-like" manual steps of genetics tests \\
\hline \multirow{4}{*}{ Inventory of Information } & Excessive batching of samples in queues \\
\hline & Creating excessive inventory of reagents that \\
\hline & $\begin{array}{l}\text { Complicated retrieval of samples or complicated access to needed } \\
\text { information }\end{array}$ \\
\hline & Poor 5 S's (messy, disorganized) laboratory or office or computer spaces \\
\hline \multirow{2}{*}{ Unnecessary Movement of People } & Unnecessary movement of people during task execution \\
\hline & People having to move long distances to access needed items \\
\hline \multirow{5}{*}{ Rework, Defects } & $\begin{array}{l}\text { Untestable samples (wrong containers, wrong amount of fluid, wrong or } \\
\text { unreadable label, wrong tubes) }\end{array}$ \\
\hline & Creating defects, failed or incorrect results \\
\hline & The killer “Re's": Retest, Rewrite, Redo... \\
\hline & Incomplete, ambiguous, or inaccurate test information \\
\hline & Inspection to catch defects \\
\hline \multirow{4}{*}{ Waste of human talent and enthusiasm } & Ignoring frontline employees' knowledge, skills, and abilities \\
\hline & The negative culture that stifles human creativity and enthusiasm \\
\hline & Blaming and shaming culture \\
\hline & Fear of making imperfections visible \\
\hline
\end{tabular}

\section{The Six Lean Principles}

The process of creating value without waste is captured into six following Lean Principles. The name of a given Principle is listed in quotes, followed by the meaning and an explanation. The effectiveness of the Lean Principles has been demonstrated in a broad range of industries and work environments, including production; engineering and systems engineering; supply chain management; finance, banking and general administration; education; healthcare, and others. Oppenheim [5] contains a review of the literature which is not repeated here.

Principle 1, "Value": Capture the value defined by the customer stakeholders, who include the patient and the provider who ordered the test. The importance of capturing test value (understanding what needs to be done) with precision, clarity, and completeness cannot be overemphasized, to avoid unnecessary rework.

Principle 2, "Value Stream": Map the value stream (plan the process) and eliminate waste. This is an important tool of Lean, which maps the current test process with all end-to-end linked tasks, control/decision nodes, and the interconnecting flows necessary to realize customer value, starting from specimen collection and ending in making the results available to the provider. During the mapping process, we identify and eliminate all NVA activities, minimize all RNVA activities, and enable the remaining activities to flow predictably and efficiently (the flow is described in Principle 3). Typically, we produce two maps, the Current State that identifies all wastes and frustrations, and Future State which is waste free and ready for implementation. 
Principle 3, "Flow": Flow the work through planned and streamlined value-adding steps of the Future State map, without stopping, batching, or idle time, unplanned rework, or backflow. Keep the samples moving through the required steps without waiting.

Principle 4, "Pull": use the Just-in-time delivery of samples to the needed instrument or work station and to the final posting of the results for both the provider and patient to see. Samples should travel in as small batches as possible. Ideally, samples should be in constant motion from the point of collection until the posting of the results and archiving.

Principle 5, "Perfection": Pursue perfection in all processes. Global competition is a brutal "race without a finish line," requiring continuous improvements of processes and products. Two features of Lean help prioritize processes for improvement: (a) making all imperfections in the workplace visible to all; and (b) prioritizing to eliminate the biggest impediments to flow and to reduce costs. Seeing problems as they appear in real time is conducive to making better decisions on corrective actions and better prioritization of improvements. When noticed early, imperfections tend to be easier and less expensive to fix; unnoticed early, they tend to grow to crisis proportions and require extensive actions to mitigate. Making imperfections visible is a motivator for applying continuous improvement in real time. The enterprise should create an effective infrastructure for capturing knowledge and lessons learned and for promoting continuous education to make each new process/step better than the last.

Principle 6, "Respect for People": Respect the people in your program. A Lean enterprise is an organization that recognizes its people are the most important resource and is one that adopts highperformance work practices. In a Lean organization, people are encouraged to identify problems and imperfections honestly and openly in real time, brainstorm root causes and corrective actions without fear, and plan effective solutions together by consensus to prevent a problem from reoccurring. When issues arise, the system is blamed and not the messengers. People must not be afraid, and management must abandon the "blaming and shaming" habits and replace them with the spirit of teamwork and "we are in it together". Experienced and knowledgeable leaders lead, motivate and mentor, but also empower frontline employees to solve problems immediately. Such an environment requires a culture of teamwork, mutual respect and trust, open and honest communication, and synergistic and cooperating relationships of all stakeholders. As stated under Principle 5, a fundamental premise of Lean is to make all imperfections visible in order to eliminate them immediately, at the source, before they move downstream and grow to crisis proportions. Employees should be rewarded for bringing system (not people!) imperfections to the attention of the stakeholders. The imperfections should never be personalized; by definition, they are system problems. But in order to enable this behavior, the Respect for People Principle must be continually pursued. Lean employees and managers know that their real competitors are not their colleagues within the same enterprise, but rather an outside clinical facility.

\section{Myths to Be Debunked About Lean}

Even though Lean is a successful and well-established work organization paradigm in healthcare, eg (3), (4), several false myths about Lean make rounds in healthcare community. Therefore, we now debunk three worst myths:

\section{In Lean 'people need roller skates' to work faster and harder}

Most emphatically: Lean does not make people work faster or harder, and it does not ask employees to wear roller skates, but it expects people to work smarter [3]. Lean is not about moving faster between the work locations; instead Lean advises to reduce the amount of walking by re-architecting spaces, and then walk at a normal ergonomic speed. In Lean, nobody needs to work harder than the ergonomic rate determined by the education, training, and experience, but the employees are expected to identify and eliminate unnecessary waiting and defects and other wastes in the processes, to give them more time for adding-value work.

\section{Lean cuts corners on quality}

Lean never cuts corners on quality; quite the opposite is true: Lean improvements inherently improve work quality [8]. Lean advises to perform all tasks truly needed to deliver quality value; but also, to pay attention to waste and eliminate waste as much as possible.

\section{Leans means "mean layoffs"}

Properly implemented Lean is an enemy of layoffs. Lean uncovers productivity reserves which are utilized for good use: improvements of processes, training and education, increasing the laboratory capacity, and better service to stakeholders ${ }^{2}$.

\section{Approach}

In 2013 the first two authors of this paper (BWO and MHK) met to discuss the advantages of Lean in healthcare. Even though Kaiser Permanente (KP) as a system is highly ranked in the US healthcare [12], MHK of KP felt that there was opportunity to improve. The laboratory was chosen as an initial site to implement Lean because of its resemblance to other industries where lean has been successfully implemented. Loyola Marymount University (LMU) at the same time was developing a new master's program in healthcare systems engineering looking for real-world applications of Lean in healthcare.

As a result, a novel approach to Lean implementation was undertaken. A graduate three-course Certificate in Lean Healthcare was created at LMU, and 10 "students" were selected from the laboratory staff, all experienced managers and directors of their respective sections of the KP regional lab. Two courses, on Lean

${ }^{2}$ The increasing number of patients enrolling into healthcare system due to the ACA and the significant shortage of medical professionals practically assures no layoffs for years to come. Also, people will never stop falling ill. 
basics and advanced Lean, were followed by a hand-on project course in the laboratory under the supervision of BWO- a faculty in systems engineering. During the project students were required to design Lean improvements and develop a set of Lean Enablers for the clinical laboratories (LEfCL).

The result was 136 LEfCL described herein. Most of the Enablers are applicable across the laboratory industry and are cited in this paper. About 15 applicable only to specific situation of KP laboratories have been omitted from the present article.

Following the approach of [7], prior to formulating the Enablers, the team formulated 46 Challenges that clinical laboratories face today. Essentially, the methodology of capturing and developing the Challenges was identical to that of the Enablers: iterative collective brainstorming and editing. Most of the Challenges deal with operational problems. Some of the Challenges deal with cultural issues which are critically important for the operational problems to be addressed effectively. Lean cannot be implemented "mechanically", addressing only the tools and processes while ignoring human factors. Again, most of the Challenges are applicable across the laboratory industry and are cited in this paper. About 15 applicable only to specific situation of KP laboratories have been omitted from the present paper. Collectively the Lean Enablers attempt to answer the Challenges; however, the relationship is one-to-many and many-to-one rather than one-to-one. Most of the Challenges apply to all clinical laboratories across the industry, and these are listed without special headings. A subgroup of the Challenges applies to specific laboratory types, and these are listed under the types.

When creating the Challenges and Lean Enablers, the team made a significant effort to apply systems thinking ("the holistic big-picture thinking") for the entire laboratory system, including not only technical operations but also people and end-to-end throughput, including sample transport from collection to posting test result. Thus, the reader will see several Enablers that focus on the significance of process standardization, visual controls, facility layouts, transport, and cleaning and organization of the workplace. A one-time improvement is not a Lean process therefore the Enablers focus on perfecting the processes through continuous improvement. The Enablers promote allocation of teams to implement and measure the success of proposed solutions, as well as building in-process quality assurance from start as a preventive measure, and not relying on final inspection, which is applied too late to avoid errors. Critical emphasis is placed on workforce development, including practices such as cross training, creating multi-skilled workforce with a continuous improvement mindset, promoting participatory decision making and good leadership at all levels, as well as abandonment of the "blaming and shaming" culture $^{3}$. LEfCL are formulated as a comprehensive set of practices, intended for future training of new hires and as reference material. As such, they include both basic and advanced practices.

\section{Statistical Analysis}

Ideally, one would wish to perform control tests measuring the effectiveness of each Lean Enabler individually, including measurements of the test turn-around-time (TAT), cost and defect reductions; capacity increase, and staff morale. The task of estimating these tangible benefits from the implementation of individual Enablers is not feasible in the busy production laboratories. Instead, we provide some aggregate benefits from the combined Lean improvements undertaken in each laboratory during the period from 2014 to 2016. Not all labs measured all the above benefits, so we quote only those measured or reliably estimated.

\section{Results}

In this section the authors use many Lean terms; these are explained in the Glossary. The Japanese words are shown in italic font.

\section{Top challenges in managing clinical laboratories}

Table 2 lists 46 challenges identified for clinical laboratories. The challenges describe typical problems that need to be eliminated. Likely, KP is not the only healthcare organization experiencing the challenges. KP has decided to publish the challenges in the spirit of improvement of professional practices across the industry. The Challenges listed herein deal only with broadly understood Lean aspects (waste and value and the flow of samples through the system) rather than the normal clinical, medical, chemical, biological, electronic or test instrument issues that are the subject of the CLS knowledge. The challenges are grouped into general types, those labeled with the first digit of 1 , applicable to the entire enterprise; and those that apply to a specific laboratory, labeled with the first digits of 2 to 6 . The latter are listed only for the laboratories that were represented in the project; but the reader should keep in mind that there are many other clinical laboratory types not covered here. The second digit is the sequential number of the given challenge in the group. The grammatical form of each challenge is a statement or observation agreed upon by a team.

Table 2: Challenges.

\begin{tabular}{|c|l|}
\hline \multicolumn{1}{|c|}{ 1. Challenges that Apply to All Laboratory Departments } \\
\hline 1.1 & Insular departments and facilities with silo culture. \\
\hline 1.2 & $\begin{array}{l}\text { Untimely dissemination of information about important process and system changes that affect all employees in a given department. Inability } \\
\text { to reach and train all employees in a timely manner with information about all important changes. }\end{array}$ \\
\hline 1.3 & Insufficient and inflexible staffing to compensate for absenteeism and for specimen workload variation. \\
\hline
\end{tabular}

${ }^{3} \mathrm{KP}$ is a union environment, with several unions represented in clinical laboratories. 


\begin{tabular}{|c|c|}
\hline 1.4 & Unclear roles, responsibility and accountability. \\
\hline 1.5 & $\begin{array}{l}\text { Lack of operational standardization amongst different medical centers that order tests. Lack of sharing and adoption of best practices within } \\
\text { the enterprise. }\end{array}$ \\
\hline 1.6 & Lack of effective handoffs and information from shift to shift. \\
\hline 1.7 & $\begin{array}{l}\text { Lack of empowered proactive management, enabled to make decisions on the program strategy, resolution and implementation. Management } \\
\text { excessively top-down, lacking good understanding of local conditions. "Sink or swim culture". }\end{array}$ \\
\hline 1.8 & $\begin{array}{l}\text { Lack of mentoring and insufficient training for newly hired managers and insufficient opportunities for continued training for middle } \\
\text { management on operations and for managing in a union environment. }\end{array}$ \\
\hline 1.9 & $\begin{array}{l}\text { Unstable, fragmented, and incapable laboratory information system, causing significant waste, delays, errors, and cultural frictions. Limited } \\
\text { functionality and expertise of IT system. }\end{array}$ \\
\hline 1.10 & Inadequate metrics, real-time data collection and dissemination. \\
\hline 1.11 & Reactive rather than proactive culture. Inadequate capture and dissemination of lessons learned. \\
\hline 1.12 & Overcrowded and poorly laid out physical spaces. \\
\hline 1.13 & Lack of incentives for union employees to accept new duties and responsibilities and to suggest improvements. \\
\hline 1.14 & $\begin{array}{l}\text { A culture of resistance to change; not receptive to progress (both management and labor). Lacking the skills and training and inadequate } \\
\text { sharing infrastructure required to succeed in the environment of rapid change in technology. }\end{array}$ \\
\hline 1.15 & Too much "blaming and shaming" culture and not enough mentoring, teamwork and positive motivation and incentives. \\
\hline 1.16 & $\begin{array}{l}\text { Lack of Lean tools throughout the laboratory system, such as Kanbans, visual controls, } 5 \text { S's, Poka Yoke error-proofing devices, root-cause analysis } \\
\text { tools to assist in streamlining operations, identification of imperfections and continuous improvements. Lack of understanding that Lean is fun } \\
\text { and a must. Lack of seeing the big picture. Education and implementation of Lean tools excessively slow. Excessive approval process, then once } \\
\text { approved, delays in implementation due to project prioritization. }\end{array}$ \\
\hline 1.17 & $\begin{array}{l}\text { Lack of governance structure for the laboratory network. Insufficient alignment and coordination of the inter-dependent elements of the } \\
\text { enterprise. Strategic vision is not shared or communicated to frontline workers in an effective way. }\end{array}$ \\
\hline 1.18 & Insufficient team skills, unproductive behavior and culture. \\
\hline 1.19 & Lack of decision transparency. Decisions made without collaborating with team members. Delay in communicating decisions. \\
\hline 1.20 & Resource limitation - too many competing projects. \\
\hline 1.21 & Shrinking operational budgets that delay innovation and implementation of new technologies. \\
\hline 1.22 & $\begin{array}{l}\text { Existing procedures do not address all small problems. Small problems tend to be ignored until they turn to major issues, which require more } \\
\text { work and resources to remediate. }\end{array}$ \\
\hline 1.23 & Lack of clarity regarding priorities. Should patient results come first, or performing projects which will benefit the lab in the long-term. \\
\hline 1.24 & Retiring CLS workforce not replaced fast enough, while the volume of work increases. \\
\hline
\end{tabular}

\section{Laboratory-Specific Challenges}

Note: the lack of Challenges under a given laboratory heading, (e.g., Chemistry and Virology) does not mean that the laboratory experiences no challenges. Rather it means that its challenges are common to other laboratories and have been listed under the general section above.

\section{Collection of Samples (Phlebotomy)}

2.1 Specimens received in the laboratory randomly placed in buckets creating the waste of sorting.

2.2 Specimens laid down in buckets rather than upright in small racks, increasing processing time and contributing to clots forming at the container caps.

2.3 Lack of Kanbans and visual tools indicating that specimens require processing or special attention.

2.4 Multiple IT systems bridged ad hoc, an obstacle to efficient communications.

\section{Transportation of Samples between Facilities}

3.1 Huge geographic service area subjected to traffic congestions creating significant variability in the transportation scheduled arrival and pick up times.

3.2 Entire transportation system lacking coherent optimization, thus extending the test throughput times unnecessarily.

3.3

Lack of reliable metrics of the transportation system, which is an obstacle to system optimization: minimizing transportation time, workload by drivers, and fuel and track costs.

\section{Sample Receiving, Processing and Distribution to Laboratory Departments}

$4.1 \quad$ "Tidal wave" of specimens received at irregular times resulting in uneven flow of work to the testing department and delays in resulting.

4.2 Often times personnel is pulled to cover other tasks while specimens keep accumulating at fast rates, causing delays and imbalance in the entire system.

4.3 Unreliable instruments, and the vendor unwilling to guarantee $24 / 7$ repairs. 


\begin{tabular}{|c|l|}
\hline 4.4 & $\begin{array}{l}\text { Multiple platforms and instrument racks used in different areas of the laboratory cause the significant waste of manual transport of samples } \\
\text { between different racks. Instruments from different vendors lack industry-wide standardization. }\end{array}$ \\
\hline 4.5 & Different testing departments use different procedures often causing confusion for staff. \\
\hline 4.6 & Updates [of laboratory information, procedures, etc.] poorly disseminated. \\
\hline 4.7 & $\begin{array}{l}\text { Excessive rate of samples received from medical centers which are improperly submitted, not test ready, with inadequate volume, in wrong } \\
\text { tubes or with wrong caps, or mislabeled. Lack of Quality Assurance at the source. }\end{array}$ \\
\hline 4.8 & Unreliable specimen tracking software and process; inadequate accountability of samples and pending samples. \\
\hline & \multicolumn{1}{|c|}{ 5. Genetics } \\
\hline 5.1 & $\begin{array}{l}\text { Manual, cumbersome, "prone to error" testing processes, more suitable for (actually a leftover from) a research setting rather than a high } \\
\text { throughput clinical laboratory. }\end{array}$ \\
\hline 5.2 & $\begin{array}{l}\text { Information about testing and patient records kept on paper. Multiple fragmented databases, no automated and integrated laboratory } \\
\text { information system. }\end{array}$ \\
\hline & \multicolumn{1}{|c|}{ 6. Bacteriology } \\
\hline 6.1 & Inadequate productivity metrics, lack of typical "one sample, one count". One specimen may have 3 or more pathogens. \\
\hline 6.2 & Lack of understanding of the criticality of leveling the flow of samples leads to wasteful storing and batching of samples. \\
\hline 6.3 & Not all staff cross trained to perform all tests/procedures. \\
\hline 6.4 & Lack of FIFO resulting, which challenges the "Auto No Growth" release. \\
\hline 6.5 & $\begin{array}{l}\text { Samples not processed FIFO. Instead they are grouped into batches of like tests for convenience of staff, in order to avoid having to remember } \\
\text { different guidelines in different tests. Lack of user-friendly visual aids/controls to assist in testing and resulting FIFO. }\end{array}$ \\
\hline
\end{tabular}

\section{Lean enablers}

The 136 Lean Enablers are displayed in Table 3, organized by the Lean Principles (Value, Value Stream, Flow, Pull, Perfection, and Respect for People summarized above in the definitions section.) In most cases they are listed without additional subheadings. In several cases when a given Enabler applies to a specific laboratory department, it is listed under the department name. The Enabler numbers consist of two digits (x.y) where " $x$ " is the Lean Principle number and " $y$ " the consecutive Enabler number under that Principle. The readers will find the clear majority of the Lean Enablers to simply represent good sense. Our collective challenge is to translate this good sense into common sense (Table 3).

Table 3: Lean Enablers.

\section{Principle 1: Value}

1.1 For all value streams in the entire laboratory network, pursue cost the shortest test throughput time with high quality as the fundamental value to both the patient and the ordering providers.

1.2 In order to keep the entire enterprise competitive and the national healthcare affordable, pursue cost reductions without jeopardizing test quality or throughput time.

\section{Principle 2: Value Stream}

2.1 Train as many employees in Lean, including value stream mapping, as possible to enable the ambitious and transformative improvement culture and reduce resistance to change.

2.2 Do not rest after one "wave" of value-stream mapping and improvements. Never stop thinking of next improvements. Incentivize creative ideas from employees.

2.3 Use the powerful value stream mapping methods to identify and eliminate waste.

2.4 Create clear and concise value stream maps and go over the entire process with staff. Engage staff in identifying wastes and streamlining the process. After improving the process, remove wasteful checks or reviews.

2.5 Use value stream mapping to proactively identify all potential risks to the test throughput and quality, and mitigate them immediately. Explanation: value stream mapping of all processes in the laboratory is useful in identification of both waste and the steps that have the potential to compromise the test throughput and quality. A proactive approach is to identify such risky steps and implement error-proofing Poka Yoke devices.

2.6 Use value stream mapping to identify and eliminate wasteful legacy tasks and practices. Explanation: This enabler embraces new technology such as automation and abolishes suboptimal legacy practices such as batch processing and manual data entry. This enabler also eliminates unnecessary steps deemed necessary in the past. An example of this is urine hemoglobin dipstick testing performed before microalbumin. Hemoglobin dipstick testing is a legacy practice that does not add value to the microalbumin testing. 


\section{Principle 3: Flow}

3.1 Empower your employees to ensure the flow of value added work. Managers are to enable the employees to enable the flow.

3.2 Eliminate mura (unevenness). Optimize the entire flow of samples, including collection and inter-facility transportation to achieve as even arrival times of samples as possible.

3.3 In order to promote the Value proposition (i.e. fastest throughput with high quality), minimize the batch sizes, strive for "just in time" processing, and level and balance the workflow throughout the system.

3.4 Treat empty instruments as a Kanban signal to load the next test samples. Keep feeding the instrument. Remember: throughput is more important than maximizing machine utilization.

3.5 Do not slow down or batch any activity "because a downstream process will not need it for hours". Example: Sparse pickup times of samples for transportation to a central laboratory should not be used as an excuse not to load the samples into transportation totes, and to batch them instead. This enabler promotes just-in-time feeding of the totes rather than batching. Then focus on improving the transportation system.

3.6 Eliminate inter-shift stoppage of testing. Enable continuous testing.

3.7 Eliminate the waste of moving samples manually between different racks. Load samples pre-analytically directly onto analyzer racks. To the extent possible, influence the instrument manufacturers to standardize all racks.

3.8 Do not schedule the maintenance of all instruments at the same time unless it is an off-work shift. During work hours, stagger the maintenance, calibration and QC so that most instruments are available for testing at any given time to promote leveled and balanced flow and maximize throughput time.

3.9 Establish the operation hours of each laboratory based on patient needs, test volume and arrival times of samples rather than on the convenience of the staff or legacy practices. Stagger start times for employees during peak hours of service to support uneven workloads. Vary the hours (of appropriate employees, if applicable) as needed to complete all testing and verify and post all results the same day that the sample arrived, if possible. Develop schedules to satisfy the flow needs, however involve employees in schedule decision making to assure maximum fairness, predictability and cooperation. In all decisions keep in mind the Value proposition.

3.10 If economically justified, automate processes where applicable to eliminate rework. E.g. move from a paper system to a paperless electronic system.

3.11 Develop and implement error proofing Poka Yoke devices and visual aids to promote error/mistake proofing and monitor error trends. Monitor the system and not individuals.

3.12 Routinely and frequently conduct huddles to promote the best flow.

3.13 Create efficient hand-off protocols, particularly between shifts and between departments. Use Lean tools such as a flow visualization to promote seamless flow of specimens for processing. Achieve smooth flow by performing the tasks right the first time. Minimize the number of handoffs.

3.14 Ensure sufficient workspace and effective layout for maximum efficiency and ergonomics.

3.15 Release test results as soon as they are available just-in-time without batching. Assure availability of staff.

3.16 Where test results need comments, and when applicable or appropriate, develop and promote standardized ("canned") comments in software for fast computerized posting of results, and train staff accordingly.

3.17 Implement effective and user-friendly local visual aids such as test guidelines for staff, to assist them in remembering test details and enable the performance of tests in the order received.

3.18 Create easy-to-access visual displays of all notifications regarding important, time sensitive, operational, and technical changes.

3.19 In order to minimize the waste of expired reagents and consumables follow the rule of FIFO. Arrange the storage areas, shelves and carriers for efficient FIFO.

3.20 Determine capacity using actual resources. Identify and eliminate bottlenecks. Until a given bottleneck is eliminated, plan for slightly less than full capacity (by about 5-10\%) to accommodate flow variability and avoid stoppages. Explanation: Using industry standards and benchmarks, if possible, the capacity of every laboratory process should be carefully estimated to avoid the wastes of mura, muda, and muri. If the laboratory workload is exceeding the capacity, then the Lean way would be to lower the volume slightly to give the laboratory time to provide additional resources for the extra work without any disruption to the operations.

3.21 Facilitate just-in-time access to information needed to execute any procedure in the department.

3.22 If economically justified, use cutting-edge technologies to obtain faster results and improve the system productivity and test quality.

3.23 Make sure that all procedures are current and capture the latest changes, and release them promptly with minimum bureaucracy as better ideas are created.

3.24 Consolidate instrument platforms (i.e. use the same instruments) to reduce maintenance, calibration and QC activities, increase productivity and maximize physical space utilization.

3.25 Standardize to single quality-control software application to eliminate variability.

3.26 Enable auto verification. Auto-release the majority of results based upon rules-based algorithms and complete the testing of all samples on the day of receipt. Promote a culture of continuous result reporting.

3.27 Maximize throughput up to instrument manufacturer's claim. 


\section{The remaining enablers address the flow in applicable individual laboratory departments.}

\section{Collection of samples}

3.28 Continually strive to catch and correct all errors in sample collection or submittal at the source. Standardize and promote best practices to assure that all samples delivered to the testing facility are "test ready". Promote "zero defects". All samples to be submitted correctly (in proper tube within specimen requirements, at the proper transportation temperature, with correct volumes and correct and readable labels).

\subsection{Expedite handling of STAT and Timed Critical specimens after being drawn.}

3.30 Provide visual cues for testing personnel when specimens with special processing and TAT requirements (STAT and Timed Critical) are required.

3.31 Where applicable, create a centralized visual board to alert staff when inpatients are not available for sample collection to minimize wasted trips for sample collection.

Explanation: Visual boards placed in a centralized department area would indicate the availability or absence of the patient in the room. Such boards would reduce or eliminate unnecessary walking to the patient's room and searching for a nurse to communicate the patient's return time. The board would allow staff members to reschedule the draw and proceed to the next available phlebotomy patient.

3.32 Use vein finding devices to reduce missed draws on the patients known for difficult phlebotomy.

3.33 Optimize the handling of specimens by placing them directly into racks for sorting and processing, and easy quality assurance.

3.34 Implement visually effective boards to monitor testing TAT and pending draws across all medical center facilities. Display STAT cases individually, and non-STAT orders cumulatively.

3.35 Use $5 \mathrm{~S}$ and implement timers with visual or audio signals to ensure that samples have been given sufficient time to clot prior to centrifugation to eliminate downstream issues such as fibrin and sample integrity problems.

3.36 Implement Computer on Wheels (COWs) or electronic tablets for front-line patient service screeners to ensure members are directed to the appropriate station promptly, and all order and payment discrepancies are resolved immediately.

3.37 Cross train phlebotomy staff to perform patient check-in to permit flexible response to varying traffic loads and during peak hours. Explanation: During peak hours, phlebotomy staff would carry dual tasks of check-in and phlebotomy. Patients would not need to wait twice for the check-in and to have their blood drawn. After checking-in the patient, the phlebotomy staff would immediately draw the patient's blood. This would reduce the wait times for the patients.

\section{Transportation}

3.38 In the entire transportation flow eliminate idle states, wait and batching as much as possible.

3.39 Eliminate long wasteful walks throughout building corridors to fetch samples. Place sample pick-up locations close to truck parking areas. Use a local runner to deliver the samples to the trucks and dedicate the drivers to driving duties.

3.4 Perform comprehensive optimization of the transportation of samples between different facilities and the regional laboratory. Use advanced modeling methods to assure the most efficient transportation times.

3.41 Implement transportation metrics which are necessary to understand and optimize transportation, such as point-to-point transportation times, gasoline use, number of drivers, hours on the road, hours idle.

3.42 After optimization, continuously monitor the transportation system to ensure that it remains effective and optimized.

\section{Sample Receiving and Initial Processing}

3.43 Strive to eliminate all waiting, idle states, batching, etc. Continuously load the pre-analytic automation equipment at all times to promote fastest throughput times.

3.44 Promote continuous flow (just-in-time) of samples by processing and sending samples to the testing facilities at shortest possible intervals, even when the transportation vehicle is not full.

3.45 Standardize analyzer racks to eliminate the wasteful activity of moving samples through multiple containers.

3.46 Encourage manufacturers to produce needed products such as blood collection tubes that use light-protected glass or plastic, and standard racks that can be used in all instruments.

3.47 Implement cost-effective real-time tracking of samples using Radio Frequency Identification (RFID). Explanation: This enabler addresses the issue of tracking patient specimens and knowing at all times where any given specimen is located. The current manual tracking system is error prone and time consuming. Specimens are lost between medical centers, testing facilities, and the processing area. Implementing real time tracking with RFID technology will eliminate this issue. It will be a passive process requiring no staff intervention or manual handling. It will record when the samples are collected, transported, tested, resulted, and then archived. Specimen location will be known at all times ensuring specimen integrity, stability, and compliance. Testing delays will be reduced, patient satisfaction improved, and treatment implemented rapidly-all creating a positive outcome for our patients. Currently the only barrier to entry is cost; however, as more laboratories adopt this technology, economies of scales would take effect and bring cost down allowing widespread adoption.

\section{Molecular Genetics and Cytogenetic Lab}

3.48 Identify the frequent manual bottlenecks, and if the root cause is not self-evident, perform root-cause analysis to improve the inefficient process. Explanation: Bottlenecks are usually caused by manual processes and quite often the reasons for bottlenecks are self-evident. When the solution provided for presumed self-evident bottleneck does not solve the problem then a more formal analysis may be required to identify the causes and apply appropriate solutions.

3.49 Use visual controls to provide information on the status and flow of testing steps. Explanation: The flow of molecular genetic testing usually contains multi-step processes, including but not limited to extraction, amplification (signal or target), and detection. Visual controls in the lab showing the realtime status of each sample would facilitate the flow and improve the throughput. 
3.50 Implement a genetics laboratory information system (GLIS) specific to genetics department, that could efficiently track the processes in all testing phases (i.e., pre-analytical, analytical, and post-analytical).

\section{Principle 4: Pull}

4.1 For maximum efficiency and continuity of the flow use visual Kanbans to coordinate the tasks along the flow and improve communications between technical and non-technical personnel.

4.2 Establish Kanbans and Minimum \& Maximum levels for all supplies.

4.3 Reduce batch sizes to single pieces (a testing instrument rack can be regarded as Lean equivalent to "single piece"), strive to have "just in time" processing as much as possible and balance incoming flow. Do not wait for full racks before inserting them into the machine.

4.4 Optimize the workflow by placing like instruments side by side. Improve "spaghetti" layout to minimize transport of specimens and human motion. Eliminate NVA steps.

4.5 Use minimum size batches and eliminate batching where possible, and implement continuous loading and flow and real time processing of samples to increase throughput.

4.6 Work that can be done today should not be left for tomorrow regardless of due date/TAT allowance.

4.7 Practice just-in-time inventory. Minimize lot number variation and over-ordering.

4.8 In order to level the flow, ensure at least hourly deliveries of samples to each testing laboratory in the same facility, regardless of partial or full trays or within-facility transportation vehicles.

4.9 Assure that all testing processes have an internal customer/next step defined until the result is posted. The steps of the testing process to be determined based on the needs of the next step in the process. Identify internal customer for every non routine task and coordinate the task details before beginning the work in order to avoid rework and disruption of flow.

4.10 Promote high level of cooperation between test performer and result reviewer, where applicable, to minimize mistakes and maximize quality.

4.11 Include only the information on test results that are needed by the physician, and eliminate unnecessary comments and information to promote clarity. Implement and use standardized "canned" phrases where appropriate.

4.12 Eliminate unnecessary storage and batching of specimens in bins and cabinets after the specimen are logged-in for testing. Proceed directly to testing.

\section{Principle 5: Perfection}

5.1 Practice continuous improvement through collaboration, cooperation, and communication between all relevant stakeholders, particularly among those affected in the process. Solicit ideas for process improvement. Continually improve all processes. Capture best practices through benchmarking and professional networking and share/communicate them broadly with other staff, and across the enterprise if applicable.

5.2 Capture verified improvement ideas into new standards efficiently. Assure efficient approval and release process to enable the improved standards to become effective ASAP.

5.3 Develop a culture in which people are not afraid to make all imperfections visible, so that the imperfections can be immediately corrected or improved. Conduct regular meetings to address them, using small teams of local stakeholders (Kaizen teams).

5.4 Take corrective action immediately when any imperfection becomes apparent, do not delay the correction process.

5.5 Strive to make each process totally predictable in terms of effort, cost, time and quality. Keep reducing variation.

5.6 Use and discuss failures as opportunities for learning, emphasizing process and not people problem.

5.7 Standardize policies, procedures and workflows throughout the enterprise. Disseminate best practices and experiences broadly.

5.8 Use simple and concise communication modes such as an A3 form throughout the enterprise.

5.9 Use well-proven Lean tools such as visual controls, standards, Kanbans, Poka-Yoke mistake proofing, 5S's workplace organization, flow leveling and balancing, spaghetti diagrams, Gemba walks, waste walk sheet, forms, work cells, as well as quality tools such as Pareto and Ishikawa diagrams, root cause analysis, 5 whys, etc., to uncover all imperfections and develop effective improvement plans.

5.10 Sign effective and well-written contracts with equipment repair contractors to minimize equipment downtime.

5.11 Provide regular feedback to the supplier of any laboratory information system (LIS) on the system performance. Provide suggestions for software improvements and request rapid implementation.

5.12 Encourage staff to continuously scrutinize each step of specimen processing, understanding its contribution to patient care and cost. Prevent the significant cost due to rejected/canceled specimens.

5.13 Prevent duplicate orders with smart software and request notification alerts.

5.14 Provide wireless communication means to inpatient staff in order to reduce unnecessary walking and improve real-time communications with nurses and physicians.

5.15 Where inefficient testing is based on a legacy research process, develop efficient and robust new production flow.

5.16 Provide simple and easy-to-understand metrics and statistical trends on process quality, throughput and other critical parameters. Share them with all employees on a regular basis; best using visual displays (e.g., TV monitors), which are visible to everyone. 
5.17 Use metrics that measure Value to monitor and improve the system and not the people. Avoid measuring individual performance for blaming and shaming. When performing root cause analyses, look at the process rather than individual employees.

5.18 Use and discuss errors/failures as opportunities for learning, emphasizing process and not people problem.

5.19 In order to break the silo culture and to optimize the flow throughout the process, empower teams to engage in efficient cross-communication and constructive exchange of ideas.

5.20 Promote "right the first time" mentality. Update and improve electronic laboratory information to contain complete, correct, up-to-date, and easy to follow information.

5.21 For facilities that use the same analyzers and test menus, create staff pools that can be shared.

5.22 Design effective standards and protocols for handling and reducing imperfect test orders and co-payment issues.

5.23 Reduce the number of approvals for projects and concurrentize the approvals.

5.24 Keep a record, preferably online, of lessons learned during test development and implementation process, and have this document accessible to all for learning and review. Assure that these documents are reviewed and absorbed before initiation of new projects.

5.25 Promote a deep and thorough failure analysis and take appropriate corrective action without creating waste of overproduction.

5.26 Provide regular feedback to suppliers of reagents and equipment on their product performance so they can improve.

5.27 Rotate employees in different areas and tasks to identify imperfections and share their experiences for processes improvement.

5.28 Promote the culture of small Kaizen teams for rapid improvement. Protect them from being assigned to other projects while they are performing the Kaizen.

5.29 Maximize opportunities for future upgrades.

5.30 Provide clear, detailed, and concise standard procedures or checklists for all test processes.

5.31 Standardize and reduce the number of software applications used for operations such as QC software, or results review and release software.

5.32 Promote hardware standards across laboratories.

a. Standardize power plugs configurations

b. Standardize power requirements of instruments

c. Automate inefficient analyzer data dumping process so that the instrument does not shut down due to lack of data storage space

d. Standardize terminology as it pertains to common laboratory components (racks, trays, etc.)

5.33 Optimize inventory management and ordering using best vendor contracts.

5.34 Schedule shift-to-shift huddles of management and staff to enable continuity, quality, and innovation between teams and shifts. Use the huddles to disseminate immediate and dynamic information and reduce inter-shift handoffs and wastes.

5.35 Utilize real-time metrics and data to display quality, throughput and other important trends.

5.36 Standardize instruments, procedures and protocols in the entire enterprise.

5.37 Use after-action reviews and lessons learned from previous projects before starting new projects.

5.38 Allocate appropriate operation budget to allow for innovation; sufficient and flexible staffing to react to absenteeism and specimen workload variation; education of exempt and non-exempt employees; and standardization of equipment and assays across the enterprise.

5.39 Ensure all stakeholders are present when implementing new technologies.

\section{Lean Enablers for Training}

5.40 Realize that training is the most effective improvement method, with immediate payback. Perform frequent training as needed for all employees in new standards and to improve quality and throughput.

5.41 Promote effective communications among all stakeholders and train accordingly.

5.42 For all repetitive work develop effective standards/procedures or checklists, train and mentor employees in the standards, and monitor progress. Facilitate acceptance and compliance with standards by involving employees in the optimization and drafting of procedures, increasing the ownership, good training and communication.

5.43 Train staff to troubleshoot routine instruments breakdowns and minor problems and routine maintenance. Mentor and share the knowledge.

5.44 Optimize labor utilization: Utilize highly-trained technical staff for complex tasks and leave simpler tasks to non-technical staff.

5.45 Promote cross training of employees to provide flexibility in operations. Standardize skill sets to enable cross-training.

5.46 Provide extensive training for newly hired laboratory management team members. Create HR Academy specific to the laboratory environment. Academy should have different levels - Entry, Mid-Level and Advanced. Establish mandatory off-site, live leadership training courses for each level of management. Although newly hired laboratory managers have experience from their previous work places, they are most probably not familiar with the new environment and culture. Thus, a newly hired manager should spend sufficient time being mentored by other managers not only in the duties to perform but also in the new culture. Such mentoring and training will be conducive to teamwork, consensus building, and will introduce clarity to the laboratory operations. 


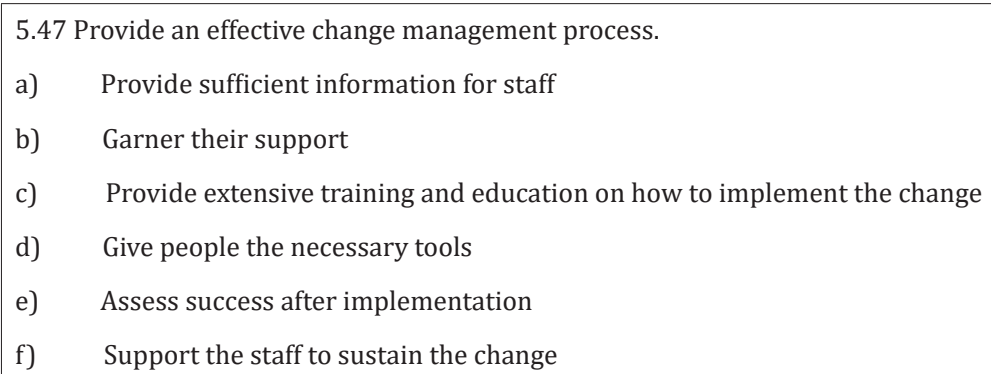

Specific Enablers for the Collection of Samples

5.48 Re-design and optimize the layout of waiting rooms and phlebotomy stations to accommodate special needs (e.g., handicapped, pediatric cases), as well as the traffic variation in time.

5.49 Install electronic visual aids such as overhead monitors to track patients' check-in and status.

5.50 Develop a robust and effective system to catch all defective and untestable samples (phlebotomy, urine, and other collected samples) at the source: wrong/missing label, insufficient quantity, wrong cap, wrong container, etc. This enabler is critical to reduce the significant waste of treasure, time and resources.

\section{Principle 6: Respect for People}

6.1 Enroll all front-line management in comprehensive Lean education. The education strongly promotes respect.

6.2 Use Lean practices to provide the work environment which is conducive to best performance and morale.

6.3 Promote a bottom up process improvement culture rather than an authoritarian top-down decision process. Empowering employees to initiate process improvements is a winning proposition for the laboratory since the employees know details of their work much better than management. The empowerment increases pride in work and changes the workforce into problem solvers. Relying only on managers for problem solving reduces buy-in and limits the number of the solvers.

6.4 Encourage managers to practice Gemba walks to see laboratory needs, system problems and risks proactively, without "blaming and shaming" the staff.

6.5 Proactively mitigate major and recurring laboratory problems such as delay in TAT, instrument breakdowns, staff shortage, contamination, etc.

6.6 Communicate and align staff in the goals of the department.

6.7 Before implementing new software, involve all stakeholders in in-depth interviews and drafting requirements and later in verifying and validating them.

6.8 Practice Gemba walks and involve staff in decision making on how to design the workflows for laboratory information system.

6.9 Promote teamwork, motivate employees, and treasure the ideas and values that they contribute.

6.10 Encourage employees to advance their career through taking on new duties and responsibilities.

6.11 Utilize modern technology such as WebEx to provide timely information when an individual employee cannot attend a given meeting in person.

6.12 Implement posting of updates on bulletin boards clearly visible to staff.

6.13 Implement updated pop-up alerts for each individual employee when the staff logs on to the system.

6.14 Use after-action reviews and lessons learned from previous programs/practices before starting new projects.

6.15 Provide necessary tools for staff/management for collection of metrics and statistics on TAT and quality. Then display the metrics and trends for all to see on monitors.

6.16 Involve staff in optimization of laboratory lay-out and flow design and in 5S activities.

\section{Numerical results of lean improvements}

Lean improvements are described in the following testing laboratories: genetics, genetic Fluorescence in Situ Hybridization (FISH), automated chemistry, bacteriology, and bacteriology ova and parasite test laboratories. In each case, we first summarize the undertaken Lean improvements in a letter-numbered form, followed by a short table with available results. We close with Lean improvements in the Specimen Processing Department (SPD), which receives samples from inter-city transportation trucks, sorts the samples, prepares them for laboratory work, loads them into racks and loads the racks onto robots for automated transport to individual laboratory departments (all labs are in the same building but on different floors and corridors). Besides the five laboratories quoted here, several others attempted Lean improvements, but staff changes prevented the capture of improvement data.

\section{Molecular Genetics Pathology Laboratory}

The initial state in 2014 was a slow, highly manual process reminiscent more of a university research lab then a production 
clinical laboratory, involving manual entries and transcriptions between MS Office tools and different computers.

\section{Lean improvements}

a. Implementation of a new Genetics Laboratory Information System (GLIS). All processes now performed in one system from beginning to the end by one technologist, eliminating hand offs and the need for multi-system workflow. Hand offs were the main cause of delays and communication failures and eliminating them was a major improvement.

b. Elimination of the manual data transfers between different files and systems, which eliminated the need for multiple reviews to assure the transfers were performed correctly.

c. Elimination of manual transcription, which wiped out the transcription errors and eliminated the need for a daily review of the data entries. Prior to Lean, regular reviews were held for every step of testing to catch the mistakes, and were not fully successful in catching transcription errors.

d. Elimination of batching and inventory, tasks not needed, idle states, rework, waiting, human motion, and material transport; reduction of effort on tasks needed.

e. The reduction of TAT for the BCR-ABL1 tests from 8 to average of 1.5 days reduced the patient wait for chemotherapy and significantly improved the patient's care and experience.

The results are shown in Table 4.

Table 4: Results from Lean Improvements in Molecular Genetic Pathology, 2014-2016.

\begin{tabular}{|c|c|c|}
\hline Parameter & $\begin{array}{c}\text { Initial State in } \\
\mathbf{2 0 1 4}\end{array}$ & $\begin{array}{c}\text { Improved State in } \\
\mathbf{2 0 1 6}\end{array}$ \\
\hline $\begin{array}{c}\text { Average Turn Around Time } \\
\text { in Molecular Genetics }\end{array}$ & 10 days & 5 days (50\% reduction) \\
\hline $\begin{array}{c}\text { Average Turn Around Time } \\
\text { for BCR-ABL1 tests }\end{array}$ & 8 days & $\begin{array}{c}1.5 \text { days }(81 \% \\
\text { reduction) }\end{array}$ \\
\hline $\begin{array}{c}\text { Cost reduction from } \\
\text { implementation of GLIS and } \\
\text { reduction of manual labor }\end{array}$ & Baseline & $\$ 250,000 / \mathrm{yr}$. \\
\hline $\begin{array}{c}\text { Cost reduction in BCR-ABL1 } \\
\text { tests }\end{array}$ & Baseline & $\$ 100,000 / \mathrm{yr}$. \\
\hline $\begin{array}{c}\text { Capacity increase without } \\
\text { staff increases }\end{array}$ & 40,000 tests/yr. & $\begin{array}{c}48,000 \text { tests } / \mathrm{yr} \text { (20\% } \\
\text { increase) }\end{array}$ \\
\hline Effect on staff morale & Baseline & $\begin{array}{c}\text { "Unbelievably high: they } \\
\text { value the significant } \\
\text { improvements in } \\
\text { workflow and patient } \\
\text { care" }\end{array}$ \\
\hline
\end{tabular}

\section{Molecular Genetics Fish Laboratory}

\section{Lean improvements}

a. Implementation of the GLIS,

b. Batch size reduction: multiple FISH probes (up to 8) dropped on one slide which reduced both the number of slides to be washed and the amount of FISH probe needed (to $1 \mu \mathrm{L}$ from $4 \mu \mathrm{L}$ ), causing a significant cost reduction.

c. The single piece flow initiated. Section managers receive cases as soon as they completed and cases are reviewed and sent for sign-out one case at a time replacing former batching.

d. Improved interfaces with medical centers allow for many of the samples to come already accessioned, which caused drastic reduction in wait times.

e. The ordering physician receives test result instantaneously after it is signed off electronically, replacing the former system in which several hours of delay occurred waiting for a technologist to first export cases into Laboratory Management System software and then separately to release them into Health Connect software before the physician would receive the result.

f. Elimination of rework: data entry into FileMaker database has been eliminated (which was a rework of data entry).

g. Better interfaces in the GLIS and the use of barcodes dramatically reduced transcription and misspelling errors of demographic and clinical information on forms. The physicians now place their own orders and enter the required information, thus eliminating the searches by laboratory staff for the information.

h. Due to the GLIS, elimination of paper folders for each FISH test, and of the need for copying of the genetic test requisition forms. The use of barcodes on slides eliminated handwritten lists.

i. Elimination of the manual flagging of STAT cases thanks to GLIS.

j. Electronic tracking and usage of the reagents before they expire.

k. Dramatic elimination of human walking with paper charts due to the electronics replacing paper.

l. Human walking reduction due to a placement of instruments much closer to the set-up area.

m. Automation of the FISH washing eliminated variability in the final slide quality and ensured consistent test reproducibility.

n. Sign offs of results performed in the evenings increased the number of test releases in a $24 \mathrm{hr}$ period. Also, employees' shifts were changed to better address work flow and reduce overtime cost.

The results are shown in Table 5.

\section{Automated Chemistry Laboratory}

\section{Lean Improvements:}

a) Samples sent hourly to the lab to reduce batching

b) "Spaghetti" layout of lab spaces streamlined

c) Samples loaded directly onto instrument racks to eliminate manual transfer of samples between different racks (on subset of instruments only) 
Table 5: Lean Improvements in FISH Laboratory, 2014-2016.

\begin{tabular}{|c|c|c|}
\hline Parameter & $\begin{array}{c}\text { Initial State in } \\
\mathbf{2 0 1 4}\end{array}$ & Improved State in 2016 \\
\hline TAT reduction & 14.7 hrs. & $10.3(30 \%)$ \\
\hline Cost reduction & Baseline & $\$ 200,000$ \\
\hline $\begin{array}{c}\text { Reduction of clerical } \\
\text { errors, } \%\end{array}$ & Baseline & $70 \%$ \\
\hline $\begin{array}{c}\text { Capacity increase with } \\
\text { minimal use of overtime } \\
\text { and no new FTE's }\end{array}$ & 8630 tests \\
\hline Effect on staff morale & Baseline & $\begin{array}{c}\text { Improved. Staff offered } \\
\text { many suggestions for the } \\
\text { listed improvement. }\end{array}$ \\
\hline
\end{tabular}

d) Originally, the daily maintenance, calibration and quality control was performed on all instruments during the morning shift, effectively idling all instruments, causing the buildup of large batches of samples, and extending the throughput times. This work was staggered so that only a third of the machines are idle per shift, and $2 / 3$ are productive, to enable continuous testing and maximize throughput. e) Batch size reduction effort in all applications and migration towards single piece flow (treating one rack as a "single piece").Distribution of received samples to instruments in real time.

f) Implementation of auto verification

g) Elimination of wasteful Hemastix testing

h) Elimination of manual uncapping of tubes with specimen

i) Limited progress towards standardization to a single tube type throughout the system.

j) Patient moving average provides a tool to continuously monitor stability and provides early detection and notification for analytical shifts not always detected by standard QC methods thereby giving only accurate patient results

k) Consolidation of tests and validation between different analyzers

The results are shown in Table 6.

Table 6: Lean Improvements in Automated Chemistry, 2014-2016.

\begin{tabular}{|c|c|c|c|}
\hline Analyzer Machine, Test & TAT reduction & Cost Reduction & Capacity Increase \\
\hline Architects, TSH & $18 \%$ & NA & \multirow{7}{*}{ NA } \\
\hline Roche Integras, $H b A 1 c$ & $39 \%$ & NA & \\
\hline $\begin{array}{l}\text { Roche Modular P, Urine } \\
\text { Microalbumin }\end{array}$ & $29 \%$ & $\$ 26,968$ & \\
\hline Beckman AU5400, Lipid Panel & $47 \%$ & NA & \\
\hline $\begin{array}{l}\text { Siemans Centaur tests moved and } \\
\text { consolidated onto Architects }\end{array}$ & $64 \%$ & NA & \\
\hline $\begin{array}{l}\text { Roche Modular E170 tests moved } \\
\text { and consolidated onto Architects }\end{array}$ & $46 \%$ & NA & \\
\hline Tube consolidation & NA & $\$ 42,000$ & \\
\hline $\begin{array}{l}\text { Total test capacity increase on all } \\
\text { analyzers, tests/year }\end{array}$ & NA & NA & $\begin{array}{c}\text { from } 17,523,596 \text { to } 18,733,729= \\
6.9 \% \text { increase }\end{array}$ \\
\hline
\end{tabular}

\section{Bacteriology Laboratory}

\section{Lean improvements}

a) Batching minimized in in-patient hospitals: Nurses instructed to send specimens through the tube system as soon as they are collected. If the tube system is down, a runner to pick up the samples from the hospital floor and deliver them for transportation to the lab.

b) Transport time reduction by building the new lab facility close to the medical centers served.

c) Batching of samples replaced with continuous workflow. As soon as the samples are received in the SPD, staff logs them for processing and delivers to the lab in 30 min intervals.

d) Elimination of waiting using laboratory automation system. Specimen identified with barcode is loaded into a sorter, moved through a conveyer belt to the processor, then into an automated incubator. Cultures can be read and resulted earlier because this system can detect clinically relevant growth quicker than with traditional incubators and manual reading.

e) Replacement of traditional biochemical method with mass spectrometer method to identify organisms. This reduced waiting for the identification from 24 hours to 32 minutes for 16 samples (about $2 \mathrm{~min} /$ sample).

f) Elimination of tasks not needed: Batches or transfer list eliminated since cultures are incubated in a "smart" incubator where each plate is assigned a unique location based on the barcode, allowing for random and rapid retrieval.

g) Elimination of double log in. The continuous workflow eliminated the need to acknowledge receipt into the ancillary department. 
h) Implementation of automated image analysis software for detection and segregation of positive from negative samples using chromo genic agar. The software has a sensitivity of $100 \%$, and a specificity of $90-96 \%$. Plates can be segregated and resulted in groups of 40 instead of individually.

i) Elimination of transfer list reduced the need for paper.

j) Elimination of human motion: specimens are loaded onto a sorter, travel through a conveyor track, and are delivered and evenly distributed into the four specimen processors used. Once processed, the specimens are returned to the same location. One loading and unloading location replaced prior set up when each processor had to be manually loaded and unloaded.

k) Stocking of drawers by each work area with necessary supplies. One person spends one hour every other day to re-stock the drawers. Minimizes the need for CLS to get up each time they run out of supply.

The results are shown in Table 7.

Table 7: Lean Improvements in Bacteriology Laboratory, 2014-2016.

\begin{tabular}{|c|c|c|}
\hline Parameter & Initial State In 2014 & Improved State In 2016 \\
\hline TAT reduction (from all in-patient hospitals at all of Med Centers) & 86.8 hrs. & Baseline \\
\hline Cost reduction & $1,429,118$ samples & 3 FTE Lab assistants reassigned \\
\hline Capacity increase & Baseline & Total elimination of manual reading errors \\
\hline Reduction of defects & Baseline & Improved and increasing. \\
\hline Effect on staff morale & & \\
\hline
\end{tabular}

\section{Bacteriology Ova and Parasite Testing Laboratory}

\section{Lean Improvements}

a) Re-scheduling of all processing to be performed on the day shift by 1-2 lab assistants under CLS supervision, eliminating the waiting for CLS during afternoon and/or night shifts.

b) Elimination of defects due to the CLS staff now supporting Lab Assistants in real time about problem specimens.

c) Conversions to the "Total-Fix" vial from former "SAF" containers eliminated non-value adding tasks of adding formalin, and centrifuge/decant steps. The Total Fix vial being free of PVA, mercury, and formalin, is greener and safer to work with. The elimination of SAF vial and other stool collection containers from inventory simplified the ordering process for the supply staff. The elimination was gradual, stocking the new type vials as old ones were used.

d) Elimination of formalin, a flammable safety hazard and replacement of carcinogen and odor-emitting Xylene with a safer and less odorous xylene substitute has increased the morale of the staff.

e) Selection of a standardized commode stool collection container, and improved patient instructions for clarity.

f) Improved collection system combined with better instructions yield better specimen to examine.

g) Significant increase in capacity allowing for significant reduction of specimen sent to outside testing facility at large cost.

The results are shown in Table 8.

Table 8: Lean Improvements in Bacteriology Ova and Parasite Laboratory, 2014-2016.

\begin{tabular}{|c|c|c|}
\hline Parameter & Initial State In 2014 & Improved State In 2016 \\
\hline TAT reduction & Over 7 days & 4.6 days (34\% reduction) \\
\hline Capacity increase & 31,023 & 33,108 specimens (7\%) \\
\hline Cost reduction & $\$ 2.52 /$ commode collection container & $\$ 0.46 /$ commode collection container (82\% reduction) \\
\hline Defect reduction & Baseline & Not measured. "Most defects and rework eliminated due to real-time support by CLS" \\
\hline
\end{tabular}

\section{SPD}

\section{Lean improvements}

a) Effort to minimize the number of untestable samples (bad or missing label, wrong container, inadequate volume of the sample, missing samples, etc.) sent from medical centers. b) Reduction of batching of samples, more JIT processing

c) Sending samples to labs hourly rather than in large multihour batches.

The results are shown in Table 9.

Table 9: Lean Improvements in SPD, 2014-2016.

\begin{tabular}{|c|c|c|}
\hline & Initial State in 2014 & Improved State in 2016 \\
\hline Throughput (specimen/yr) & $14,000,000$ & $16,000,000(14 \%$ increase $)$ \\
\hline
\end{tabular}


Note: the increase in the numbers of specimen reflects the increase in the number of patients served, however, the increase was possible thanks to the Lean improvements. Also, note that many specimens are aliquoted into several others, so the number of tests is several times larger.

\section{Conclusion and Discussion}

The Lean Enablers for Clinical Laboratories (LEfCL) represent a collection of best productivity practices based on Lean Thinking that is minimizing waste and promoting value. The value is defined as the correct and accurate test with test results delivered to the ordering provider in the minimum time, at minimum cost, with maximum quality and improvement of staff morale. This paper presented 136 LEfCLs. The paper also presented the productivity and quality Challenges observed in clinical laboratories, which the LEfCLs remedy. Numerical results from five laboratories and from the Specimen Processing Department that serves all laboratories are quoted for the period of 2014 to 2016. Quoted are overall simultaneous improvements achieved from implementation of selected LEfCLs, including reduction in test turn-around-time, laboratory capacity increase, cost reduction, defect reduction, and staff morale improvements; however, not all these parameters were measured in all laboratories quoted. The two-year period coincided with a $14 \%$ increase in the number of tests performed due to the ACA-driven increased number of patients. Given the pressures to perform significantly more tests with minimal or no staff increases and even some staff reductions pushed the desired task of keeping accurate productivity metrics to a lower priority, so we can quote only aggregate results. Nevertheless, the Lean improvements shown vary from $6 \%$ to $81 \%$. The undertaken improvements represent a small subset of the LEfCL. Therefore, the results quoted can be regarded as highly conservative estimates of the power of LEfCL. These results suggest that Lean can be a powerful paradigm for productivity, quality and staff morale improvements in clinical laboratories as it is in other healthcare operations, and that LEfCL represent a powerful improvement tool.

In order to achieve the goals set forth by the Lean Enablers one needs to have an efficient and timely process to introduce and implement the changes. In other words, a change process management is needed that will minimize resistance and create desirable results. The Lean Enablers proposed in the "Respect for People" section are intended to facilitate the change process.

The Lean Enablers should be particularly useful for planning new laboratories and training new hires. As Covey [13] stated one of the habits of highly effective people is to "begin with the end in mind." The Lean Enablers support this goal two-fold, by stressing the need for a clear understanding of the customer (provider who ordered the test and the patient) requirements and value perception, as well as proposing various effective laboratory management practices to efficiently fulfill these requirements. Lean Thinking can be ingrained in the laboratory "DNA" at the foundation level across all the people from the time they begin as team members. The benefits of this are that the people within the organization evolve to think in Lean terms and pursue Lean as a means by which the company delivers value to its customers. In organizations of this nature, Lean simply becomes the preferred way of working, and Lean Enablers become more of an automatic response by the people doing work for their customers on a daily basis, [7].

As stated earlier, this paper provides reference material. The Lean Enablers are not intended to serve as mandatory practices, but rather as both comprehensive training materials and as a checklist of best practices that can help with managing clinical laboratories more effectively. The Enablers have been collected and organized by highly experienced laboratory managers, from the highly ranked healthcare organization, therefore ought to represent a degree of wisdom to emulate by professionals engaged in clinical laboratory work.

One should not attempt to implement all Enablers at once; that would be a formidable task. Instead, the focus should be on those that will have the highest impact, and on the "low hanging fruit". Usually the highest priorities for improvements are: the biggest impediments to flow, impediments to test quality, and the sources of providers' complaints. Line staff should be encouraged to implement the Enablers which deal with the individual's work and do not need any significant coordination with others.

Oehmen [7] stated: “...a proactive way to utilize and implement the Lean Enablers is when an organization is operating without any major difficulties, but decides to find even better ways to provide greater value to their customers. Triggers can be the strategic planning of the value stream and then choosing to proactively improve some key processes that are operating well enough in the current state. Questions, such as 'what are our theoretical limits of performance?' or 'how can we sustainably outcompete our competitors?' or 'what does true success for our customer really look like?' are asked. Great levels of success are guaranteed when an organization attains world class business performance and sets the standard for everyone else."

The intent of the Lean Enablers must be clearly understood by those who will use this information. Most importantly, the laboratory leadership must understand the six Lean Principles. The maturity of an organization's Lean understanding will help determine the customizing and tailoring required for specific situations and the laboratory management leadership.

There are some limitations to this paper. We did not have a matched control group and did not completely analyze all aspects of the lab operation but merely measured what we could while operationalizing Lean. We cannot guarantee that our results would be generalizable to laboratories of different size or scope or those not part of an integrated delivery system. However, Lean has been applied across a wide range of industries and there is no reason to think that at least directionally our results would not generalize. Lastly, much of Lean implementation requires good leadership and the right culture.

To quote from [7]: "Much of the success of all Lean deployment truly rests with the quality of the Leadership of the organization. 
Leaders of the organization should define what their approach is, communicate it with great repetition, visibly participate with the Lean transformation activities, and provide reward and encouragement to those who are advancing the organization's
Lean journey. Given this level of leadership support, these differing approaches become complementary and ultimately begin to achieve a Lean culture that is continuously improving itself through the implementation of Lean in the unending pursuit of perfection."

\section{Glossary (Japanese words are italicized)}

\begin{tabular}{|c|c|}
\hline $5 S^{\prime} s$ & $\begin{array}{l}\text { Workplace cleaning and organization method that includes five steps (sorting, designating } \\
\text { places, cleaning, standardization, and sustainment) }\end{array}$ \\
\hline 5 whys & A root-cause finding technique \\
\hline A3 & A single-sheet form for efficient capture of important information and problem resolution \\
\hline Balancing & Smoothing of workloads among work stations \\
\hline BCR-ABL1 & name of a test in recombinant cell lines \\
\hline CLS & Clinical Laboratory Staff (qualified technician) \\
\hline COWS & Computer on Wheels \\
\hline DNA & Deoxyribonucleic acid (the hereditary material in humans and almost all other organisms). \\
\hline & Here used colloquially meaning "the nature of". \\
\hline FIFO & First In/First Out \\
\hline FISH & Fluorescence In Situ Hybridization \\
\hline FTE & Full Time Equivalent \\
\hline Gemba (walk) & $\begin{array}{l}\text { Observing first-hand the front-line work activities where the action is taking place in order to } \\
\text { see opportunities for improvement, assist staff, proactively mitigate risks and problems, and } \\
\text { solve issues. }\end{array}$ \\
\hline GLIS & Genetics Laboratory Information System \\
\hline Ishikawa diagram & Diagram that shows the specific causes of a certain event or problem \\
\hline JIT & $\begin{array}{l}\text { Just-in-time; Production organizations in which items are processed and created in single } \\
\text { pieces to meet demand, rather than in surplus or in advance of the need. }\end{array}$ \\
\hline Kaizen & Rapid improvement team/processes, typically by a small group of local stakeholders \\
\hline Kanban & Visual signal or card signaling need to refill or to move the item to the next step \\
\hline KP & Kaiser Permanente \\
\hline LDT & Laboratory Developed Tests \\
\hline Lean & Creating best value with minimum waste \\
\hline Leveling & Smoothing out the incoming orders for better utilization of resources \\
\hline LIS & Laboratory Information System \\
\hline LMU & Loyola Marymount University in Los Angeles \\
\hline MC & Medical Center \\
\hline МOB & Medical Office Building \\
\hline Muda & Waste, no value added \\
\hline Mura & Unevenness or irregularity, inconsistency \\
\hline Muri & Overburden, unnecessary stress to employees and processes \\
\hline NVA & Non-value added \\
\hline Poka Yoke & Mistake proofing hardware or software device or alarm \\
\hline QA & Quality Assurance \\
\hline QC & Quality Control \\
\hline Pareto & Diagram which is a histogram arranged in ascending or descending order \\
\hline PVA & Polyvinyl Alcohol \\
\hline RFID & Radio Frequency Identification \\
\hline Spaghetti diagram & A diagram with traces of people or material movements used to streamline processes \\
\hline
\end{tabular}


SAF

SPD

STAT

TAT

TSH

Visual controls

VSM

Waste walk

Work cell

\section{Institution}

\section{Kaiser Permanente}

\section{References}

1. (2014) PCAST (President's Council of Advisors on Science and Technology), Report to the president better health care and lower costs: accelerating improvement through systems engineering. executive office of the president, USA.

2. (2014) CBA (Congressional Budget Office), Updated estimates of the effects of the insurance coverage provisions of the affordable care act.

3. Graban M (2011) Lean Hospitals: Improving Quality, Patient Safety, and Employee Engagement. ( $2^{\text {nd }}$ edn), CRC Press, Boca Raton, Florida, USA.

4. Toussaint J (2010) On the Mend: Revolutionizing Healthcare to Save Lives and Transform the Industry. Lean Enterprise Institute, Boston, USA.

5. Oppenheim BW (2011) Lean for systems engineering with lean enablers for systems engineering, Wiley, Hoboken, New Jersey, USA, pp. 336.
6. Oppenheim BW, Murman EM, Secor DA (2011) Lean Enablers for Systems Engineering. J Systems Engineering 14(1): 29-55.

7. Oehmen J (2012) The Guide to Lean Enablers for Managing Engineering Programs. PMI-INCOSE-MIT LAI.

8. Womack J, Jones D (2003) Lean Thinking: Banish Waste and Create Wealth in Your Corporation, ( $2^{\text {nd }}$ edn), Simon \& Schuster, New York, USA.

9. Ohno $\mathrm{T}$ (2014) Workplace Management: Special (100 ${ }^{\text {th }}$ Birthday). McGraw-Hill, USA, Dec. 11, 201213.

10. Oppenheim BW (2014) Lean Enablers for Clinical Laboratories, Kaiser Permanente Report, USA.

11. Liker J (2004) The Toyota Way: 14 Management Principles from the World's Greatest Manufacturer, McGraw-Hill, New York, USA.

12. Consumer Reports (2015).

13. Covey SR (2002) The 7 Habits of Highly Effective People. Stephen covey. com Publication. 